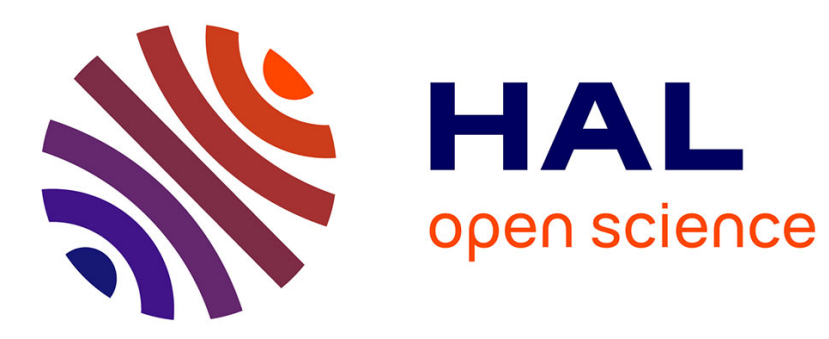

\title{
Inverse problem and concentration method of a continuous-in-time financial model
}

Tarik Chakkour, Emmanuel Frénod

\section{To cite this version:}

Tarik Chakkour, Emmanuel Frénod. Inverse problem and concentration method of a continuous-in-time financial model. International Journal of Financial Engineering, 2016, 10.1142/S242478631650016X . hal-01359689

\section{HAL Id: hal-01359689 \\ https://hal.science/hal-01359689}

Submitted on 2 Sep 2016

HAL is a multi-disciplinary open access archive for the deposit and dissemination of scientific research documents, whether they are published or not. The documents may come from teaching and research institutions in France or abroad, or from public or private research centers.
L'archive ouverte pluridisciplinaire HAL, est destinée au dépôt et à la diffusion de documents scientifiques de niveau recherche, publiés ou non, émanant des établissements d'enseignement et de recherche français ou étrangers, des laboratoires publics ou privés. 


\title{
Inverse problem and concentration method of a continuous-in-time financial model *
}

\author{
Tarik Chakkour, Emmanuel Frénod \\ Univ. Bretagne-Sud, UMR 6205, LMBA, F-56000 Vannes, France
}

July 15, 2016

\begin{abstract}
In a continuous-in-time model there is the important financial quantity called Loan which can not be determined directly in terms of Algebraic Spending but has a major impact on the financial strategy. In this paper, we use a mathematical framework to discuss an inverse problem of determining the implied Loan Measure from Algebraic Spending Measure when it is possible. In addition, we build a numerical method to concentrate a measure as a sum of Dirac masses.
\end{abstract}

\section{Contents}

1 Introduction 2

2 Continuous-in-time financial model 2

2.1 The Radon measure space . . . . . . . . . . . . . . . . . . . . . . . . . 2

2.2 Financial quantities of the model $\ldots \ldots \ldots \ldots \ldots \ldots \ldots$

3 Inverse problem of the model $\quad 4$

4 A concentration method of the model $\quad 10$

4.1 Construction of the partition of unity $\ldots \ldots \ldots \ldots \ldots \ldots \ldots \ldots$

4.1 .1 Initialization step . . . . . . . . . . . . . . . . . . . 11

4.1 .2 Iteration step . . . . . . . . . . . . . . . . . . . . . . . 12

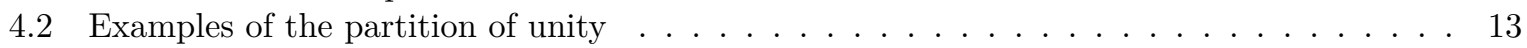

4.3 Financial simulation . . . . . . . . . . . . . . . . . . . . . . . 14

${ }^{*}$ This work is jointly funded by MGDIS company (http://www.mgdis.fr/) and the LMBA (http://www.lmba-math.fr/). 


\section{Introduction}

The continuous-in-time financial model, first introduced by Sundaresan in [9], constitutes a powerful tool for studying the development of continuous-in-time methods in finance. These include computational and estimation methods to test and implement continuous-time models. We refer to papers $[1,8]$, which are dealing with continuous-in-time financial model.

At the same time, inverse problems began to be studied and applied systematically in various branches of modern science as in finance. Studying problems can be classified as inverse, and they are among the most complicated ones (since they are unstable and usually nonlinear). The reason is that solutions to inverse problems describe important properties of variable under study such as density or measure and the integrability property. Many studies have used an inverse problem in finance. For example the theory of inverse problems via Tikhonov regularisation in [2] is used in order to analyse the procedure for determining volatility in the model of Black and Scholes. H. Egger, H.W. Engl [3] study the inverse problem of option pricing under consideration and they prove a stable solution of the inverse problem and its convergence.

In recent paper [4] we have also constructed a continuous-in-time model which is designed to be used for the finances of public institutions and not for the financial market. This model permit to set out annual and multiyear budgets for any organization and describes working of loan and repayment in order to forecast its future financial plans. We build this model because of the default of a discrete model which is using tables. Indeed, we justified in the introduction of [4] why that we created this model. This model relies on measure theory and uses the mathematical tools such convolution and integration, etc. In addition, we check the mathematical consistency of this model in [4]. Frénod \& Safa [5, 7, 6] use this model in the framework of the control theory in order to set out an optimal control method for the strategy elaboration phase to better adjust the project implementation.

In a continuous-in-time model an Algebraic Spending takes into account the spending and the income to satisfy the current needs. There exists a mathematical operator that links between Algebraic Spending and Loan. If this operator is not invertible, solutions of the posed inversion problem can be brought.

The aim of the present paper is to study the inverse problem with giving a rigorous mathematical formulation to determine Loan from the Algebraic Spending and to build a concentration method in order to concentrate a measure at positions.

The paper is organized as follows. We begin by describing in section 2 a continuous-in-time model. In section 3, we study the inverse problem that link between financial quantities in the model. We analyse it on measure space in order to show its consistency. In section 4, we introduce a numerical approach which is allowing to concentrate any measure as a combinaison of Dirac measures.

\section{Continuous-in-time financial model}

In this section we recall the variables used in the model and introduced in [4] in order to describe links existing between them and their meanings. These variables are defined on the set of Radon measures.

\subsection{The Radon measure space}

We build the continuous-in-time financial model on the Radon measure space $\mathcal{M}\left(\left[t_{\mathrm{I}}, \Theta_{\max }\right]\right)$ which is a continuous and linear form acting on continuous functions space $\mathcal{C}_{c}^{o}\left(\left[t_{\mathrm{I}}, \Theta_{\max }\right]\right)$ defined over a time interval $\left[t_{\mathrm{I}}, \Theta_{\max }\right]$. The usual norm on $\mathcal{M}\left(\left[t_{\mathrm{I}}, \Theta_{\max }\right]\right)$ is:

$$
\|\mu\|_{\mathcal{M}\left(\left(t_{\mathrm{I}}, \Theta_{\max }\right)\right)}=\sup _{\psi \in \mathcal{C}_{c}^{o}\left(\left[t_{\mathrm{I}}, \Theta_{\max }\right]\right), \psi \neq 0}\left\{\frac{\langle\mu, \psi\rangle}{\|\psi\|_{L^{\infty}\left(\left[t_{\mathrm{I}}, \Theta_{\max }\right]\right)}}\right\},
$$

where \|\|$_{L^{\infty}\left(\left[t_{\mathrm{I}}, \Theta_{\max }\right]\right)}$ is the usual norm on $\mathcal{C}_{c}^{o}\left(\left[t_{\mathrm{I}}, \Theta_{\max }\right]\right)$ defined as: 


$$
\|\psi\|_{L^{\infty}\left(\left[t_{\mathrm{I}}, \Theta_{\max }\right]\right)}=\sup _{t \in\left[t_{\mathrm{I}}, \Theta_{\max }\right]}\{|\psi(t)|\} .
$$

We notice that we build the model on a Banach space $\mathcal{M}\left(\left[t_{\mathrm{I}}, \Theta_{\text {max }}\right]\right)$ in order to use the convolution and Fourier Transform.

\section{$2.2 \quad$ Financial quantities of the model}

We explained in [4] the financial quantities that are involved in the models and the relations between them. For instance the Loan Measure $\tilde{\kappa}_{E}$ and the Repayment Measure $\tilde{\rho}_{\mathcal{K}}$ are connected by a convolution operator:

$$
\tilde{\rho}_{\mathcal{K}}=\tilde{\kappa}_{E} \star \tilde{\gamma}
$$

where the Repayment Pattern Measure $\tilde{\gamma}$ is a non-negative measure with total mass which equals $1, \tilde{\gamma}$ has to satisfies:

$$
\int_{-\infty}^{+\infty} \tilde{\gamma}=1
$$

We denote by $\mathcal{K}_{R D}$ the Debt Field, given as the solution to the following Ordinary Differential Equation:

$$
d \mathcal{K}_{R D}=\tilde{\kappa}_{E}-\tilde{\rho}_{\mathcal{K}}-\tilde{\rho}_{\mathcal{K}}^{\mathrm{I}},
$$

where measure $\tilde{\rho}_{\mathcal{K}}^{\mathrm{I}}$ is the repayment of the Current Debt $\mathcal{K}_{R D}$ at initial time $t_{\mathrm{I}}$. It is called initial Debt Repayment Scheme. Initial condition for equation (5) is given by:

$$
\int_{t_{\mathrm{I}}}^{+\infty} \tilde{\rho}_{\mathcal{K}}^{\mathrm{I}}=\mathcal{K}_{R D}\left(t_{\mathrm{I}}\right)
$$

The solution of this ODE is expressed:

$$
\mathcal{K}_{R D}(t)=\mathcal{K}_{R D}\left(t_{\mathrm{I}}\right)+\int_{t_{\mathrm{I}}}^{t} \tilde{\kappa}_{E}-\int_{t_{\mathrm{I}}}^{t} \tilde{\rho}_{\mathcal{K}}-\int_{t_{\mathrm{I}}}^{t} \tilde{\rho}_{\mathcal{K}}^{\mathrm{I}}=\int_{t_{\mathrm{I}}}^{t} \tilde{\kappa}_{E}-\int_{t_{\mathrm{I}}}^{t} \tilde{\rho}_{\mathcal{K}}+\int_{t}^{+\infty} \tilde{\rho}_{\mathcal{K}}^{\mathrm{I}} .
$$

We denote by $\tilde{\rho}_{\mathcal{I}}$ the Interest Payment Measure defined by:

$$
\tilde{\rho}_{\mathcal{I}}=\alpha \mathcal{K}_{R D}(t) d t
$$

where $\alpha$ is the floating rate interest. Algebraic Spending Measure $\tilde{\sigma}$ is defined such that the difference between spendings and incomes required to satisfy the current needs. Algebraic Spending Measure $\tilde{\sigma}$ takes into account the spending and the income and it is given by:

$$
\tilde{\sigma}=\tilde{\sigma}_{g}+\tilde{\beta},
$$

where $\tilde{\sigma}_{g}$ is the Current Spending Measure and $\tilde{\beta}$ is the Measure of Isolated Spending. The balance equation between incomes and spendings is expressed by the following equality:

$$
\tilde{\kappa}_{E}=\tilde{\sigma}+\tilde{\rho}_{\mathcal{K}}+\tilde{\rho}_{\mathcal{K}}^{\mathrm{I}}+\tilde{\rho}_{\mathcal{I}} .
$$




\section{Inverse problem of the model}

In this section we inverse operators given below which are useful of the model. We study this inversibility in measure space. For that, we consider a positive number $\Theta_{\gamma}$ such that $\Theta_{\gamma}<\Theta_{\max }-t_{\mathrm{I}}$ and we set the Repayment Pattern Measure $\tilde{\gamma}$ such that:

$$
\tilde{\gamma} \in \mathcal{M}\left(\left[0, \Theta_{\gamma}\right]\right)
$$

In order that Repayment Measure $\tilde{\rho}_{\mathcal{K}}$ given by a convolution operator in relation (3) has its support in $\left[t_{\mathrm{I}}, \Theta_{\max }\right]$, we need to choose Loan Measure $\tilde{\kappa}_{E}$ with support being included in $\left[t_{\mathrm{I}}, \Theta_{\max }-\Theta_{\gamma}\right]$. Indeed, the support of convolution of two compactly supported measures $\tilde{\kappa}_{E}$ and $\tilde{\gamma}$ is included in the sum of their supports which is translated by following relation

$$
\operatorname{Supp}\left(\tilde{\kappa}_{E} \star \tilde{\gamma}\right) \subset \operatorname{Supp}\left(\tilde{\kappa}_{E}\right)+\operatorname{Supp}(\tilde{\gamma}) .
$$

Theorem 3.1. If Repayment Pattern Measure $\tilde{\gamma}$ satisfies relation (11) and if Loan Measure $\tilde{\kappa}_{E}$ is in $\mathcal{M}\left(\left[t_{\mathrm{I}}, \Theta_{\max }-\Theta_{\gamma}\right]\right)$ and Initial Debt Repayment Measure $\tilde{\rho}_{\mathcal{K}}^{\mathrm{I}}$ is in $\mathcal{M}\left(\left[t_{\mathrm{I}}, \Theta_{\max }\right]\right)$, then Algebraic Spending Measure $\tilde{\sigma}$ is also in $\mathcal{M}\left(\left[t_{\mathrm{I}}, \Theta_{\max }\right]\right)$ and has the next expression in terms of Loan Measure $\tilde{\kappa}_{E}$ :

$$
\tilde{\sigma}=\tilde{\kappa}_{E}-\tilde{\kappa}_{E} \star \tilde{\gamma}-\alpha\left\langle\tilde{\kappa}_{E}-\tilde{\kappa}_{E} \star \tilde{\gamma}, \mathbb{1}_{\mid\left[t_{I}, t \mid\right.}\right\rangle d t-\alpha\left\langle\tilde{\rho}_{\mathcal{K}}^{\mathrm{I}}, \mathbb{1}_{\mid\left[t, \Theta_{\max } \mid\right.}\right\rangle d t-\tilde{\rho}_{\mathcal{K}}^{\mathrm{I}}
$$

Let $\mathcal{L}$ be a linear operator defined from $\mathcal{M}\left(\left[t_{\mathrm{I}}, \Theta_{\max }-\Theta_{\gamma}\right]\right)$ to $\mathcal{M}\left(\left[t_{\mathrm{I}}, \Theta_{\max }\right]\right)$ acting on Loan Measure $\tilde{\kappa}_{E}$ by

$$
\mathcal{L}\left[\tilde{\kappa}_{E}\right]=\tilde{\kappa}_{E}-\tilde{\kappa}_{E} \star \tilde{\gamma}-\alpha\left\langle\tilde{\kappa}_{E}-\tilde{\kappa}_{E} \star \tilde{\gamma}, \mathbb{1}_{\mid\left[t_{I}, t[\right.}\right\rangle d t
$$

where $\left\langle\tilde{\kappa}_{E}-\tilde{\kappa}_{E} \star \tilde{\gamma}, \mathbb{1}_{\mid\left[t_{\mathrm{I}}, t[\right.}\right\rangle d t$ is density measure which density stands for the function

$$
t \mapsto \int_{t_{\mathrm{I}}}^{t} \tilde{\kappa}_{E}-\tilde{\kappa}_{E} \star \tilde{\gamma}
$$

Let $\mathcal{D}$ be an operator defined in $\mathcal{M}\left(\left[t_{\mathrm{I}}, \Theta_{\text {max }}\right]\right)$ acting on Initial Debt Repayment Measure $\tilde{\rho}_{\mathcal{K}}^{\mathrm{I}}$ by

$$
\mathcal{D}\left[\tilde{\rho}_{\mathcal{K}}^{\mathrm{I}}\right]=-\alpha\left\langle\tilde{\rho}_{\mathcal{K}}^{\mathrm{I}}, \mathbb{1}_{\mid\left[t, \Theta_{\max }[\right.}\right\rangle d t-\tilde{\rho}_{\mathcal{K}}^{\mathrm{I}}
$$

where $\left\langle\tilde{\rho}_{\mathcal{K}}^{\mathrm{I}}, \mathbb{1}_{\mid\left[t, \Theta_{\max }[\right.}\right\rangle d t$ is density measure which density stands for the function

$$
t \mapsto \int_{t}^{\Theta_{\max }} \tilde{\rho}_{\mathcal{K}}^{\mathrm{I}} .
$$

Expression (13) of Measure $\tilde{\sigma}$ can be written as follows:

$$
\tilde{\sigma}=\mathcal{L}\left[\tilde{\kappa}_{E}\right]+\mathcal{D}\left[\tilde{\rho}_{\mathcal{K}}^{\mathrm{I}}\right]
$$

We define a density measure $\tilde{\mathrm{e}}_{\alpha}$ with its density $\mathrm{e}_{\alpha}$ is exponential function of coefficient $\alpha$, i.e $\tilde{\mathrm{e}}_{\alpha}=\mathrm{e}_{\alpha}(t) d t$, where $\mathrm{e}_{\alpha}(t)=\mathrm{e}^{\alpha t}$.

Lemma 3.2. If Loan Measure $\tilde{\kappa}_{E}$ is in $\mathcal{M}\left(\left[t_{\mathrm{I}}, \Theta_{\max }-\Theta_{\gamma}\right]\right)$ and if $\tilde{\gamma}$ satisfies relation (11), then we have the following equality:

$$
(1-\mathcal{F}(\tilde{\gamma})) \mathcal{F}\left(\tilde{\kappa}_{E}\right)=\mathcal{F}\left(\mathcal{L}\left[\tilde{\kappa}_{E}\right]+\alpha \tilde{\mathrm{e}}_{\alpha}\left\langle\mathcal{L}\left[\tilde{\kappa}_{E}\right], \mathrm{e}_{-\alpha \mid\left[t_{1}, t[\right.}\right\rangle\right)
$$

where $\mathcal{F}$ stands for the Fourier Transform Operator, where operator $\mathcal{L}$ is defined by (14). 
Proof. We have the following equality:

$$
\left\langle\tilde{\kappa}_{E}-\tilde{\kappa}_{E} \star \tilde{\gamma}, \mathbb{1}_{\mid\left[t_{\mathrm{I}}, t[\right.}\right\rangle d t=\tilde{\mathrm{e}}_{\alpha}\left\langle\tilde{\kappa}_{E}-\tilde{\kappa}_{E} \star \tilde{\gamma}-\alpha\left\langle\tilde{\kappa}_{E}-\tilde{\kappa}_{E} \star \tilde{\gamma}, \mathbb{1}_{\mid\left[t_{\mathrm{I}}, s[\right.}\right\rangle d s, \mathrm{e}_{-\alpha \mid\left[t_{\mathrm{I}}, t[\right.}\right\rangle .
$$

Indeed, relation (20) is obtained using the integration by parts:

$$
\left\langle\left\langle\tilde{\kappa}_{E}-\tilde{\kappa}_{E} \star \tilde{\gamma}, \alpha \mathrm{e}_{\alpha \mid\left[t_{\mathrm{I}}, s[\right.}\right\rangle d s, \mathrm{e}_{-\alpha \mid\left[t_{\mathrm{I}}, t[\right.}\right\rangle d t=\tilde{\mathrm{e}}_{\alpha}\left\langle\tilde{\kappa}_{E}-\tilde{\kappa}_{E} \star \tilde{\gamma}, \mathrm{e}_{-\alpha \mid\left[t_{\mathrm{I}}, t[\right.}\right\rangle-\left\langle\tilde{\kappa}_{E}-\tilde{\kappa}_{E} \star \tilde{\gamma}, \mathbb{1}_{\mid\left[t_{\mathrm{I}}, t[\right.}\right\rangle d t .
$$

Using definition (14) of operator $\mathcal{L}$, equality in (20) is multiplied by $\alpha$ to give:

$$
\left\langle\tilde{\kappa}_{E}-\tilde{\kappa}_{E} \star \tilde{\gamma}, \alpha \mathbb{1}_{\mid\left[t_{\mathrm{I}}, t[\right.}\right\rangle d t=\alpha \tilde{\mathrm{e}}_{\alpha}\left\langle\mathcal{L}\left[\tilde{\kappa}_{E}\right], \mathrm{e}_{-\alpha \mid\left[t_{\mathrm{I}}, t\right]}\right\rangle
$$

Replacing $\left\langle\tilde{\kappa}_{E}-\tilde{\kappa}_{E} \star \tilde{\gamma}, \alpha \mathbb{1}_{\mid\left[t_{\mathrm{I}}, t[\right.}\right\rangle d t$ in relation $(22)$ by $\tilde{\kappa}_{E}-\tilde{\kappa}_{E} \star \tilde{\gamma}-\mathcal{L}\left[\tilde{\kappa}_{E}\right]$, we obtain the following equality:

$$
\tilde{\kappa}_{E}-\tilde{\kappa}_{E} \star \tilde{\gamma}=\mathcal{L}\left[\tilde{\kappa}_{E}\right]+\alpha \tilde{\mathrm{e}}_{\alpha}\left\langle\mathcal{L}\left[\tilde{\kappa}_{E}\right], \mathrm{e}_{-\alpha \mid\left[t_{\mathrm{I}}, t[\right.}\right\rangle .
$$

Equality (19) is obtained with applying Fourier Transform to relation (23). Consequently, the lemma is proved.

Theorem 3.3. If Repayment Pattern Measure $\tilde{\gamma}$ is satisfying relation (11) and following relation

$$
\exists \epsilon>0, \frac{1}{1-\mathcal{F}(\tilde{\gamma})} \in \mathbb{L}^{\infty}((-\infty,-\epsilon) \cup(\epsilon,+\infty)),
$$

and if Initial Debt Repayment Measure $\tilde{\rho}_{\mathcal{K}}^{\mathrm{I}}$ and Algebraic Spending Measure $\tilde{\sigma}$ are in $\mathcal{M}\left(\left[t_{\mathrm{I}}, \Theta_{\max }\right]\right)$ and satisfy the following equality:

$$
\left\langle\tilde{\sigma}-\mathcal{D}\left[\tilde{\rho}_{\mathcal{K}}^{\mathrm{I}}\right]+\alpha \tilde{\mathrm{e}}_{\alpha}\left\langle\tilde{\sigma}-\mathcal{D}\left[\tilde{\rho}_{\mathcal{K}}^{\mathrm{I}}\right], \mathrm{e}_{-\alpha \mid\left[t_{\mathrm{I}}, t[\right.}\right\rangle, \mathbb{1}_{\mid\left[t_{\mathrm{I}}, \Theta_{\max }[\right.}\right\rangle=0,
$$

then, there exists an unique Loan Measure $\tilde{\kappa}_{E}$ in $\mathcal{M}\left(\left[t_{\mathrm{I}}, \Theta_{\max }-\Theta_{\gamma}\right]\right)$ (if it exists) given by following formula:

$$
\tilde{\kappa}_{E}=\mathcal{F}^{-1}\left(\frac{\mathcal{F}\left(\tilde{\sigma}-\mathcal{D}\left[\tilde{\rho}_{\mathcal{K}}^{\mathrm{I}}\right]+\alpha \tilde{\mathrm{e}}_{\alpha}\left\langle\tilde{\sigma}-\mathcal{D}\left[\tilde{\rho}_{\mathcal{K}}^{\mathrm{I}}\right], \mathrm{e}_{-\alpha \mid\left[t_{\mathrm{I}}, t[\right.}\right\rangle\right)}{1-\mathcal{F}(\tilde{\gamma})}\right)
$$

such that (18) holds.

In order to prove this theorem, we need the following lemmas:

Lemma 3.4. If Repayment Pattern Measure $\tilde{\gamma}$ is different from $\delta_{0}$ satisfying relation (11), then, linear operator $\mathcal{L}$ given in relation (14) is a one-to-one application.

Proof. According to (23), if Loan Measure $\tilde{\kappa}_{E}$ is in $\operatorname{Ker}(\mathcal{L})$, then, we get the following equation:

$$
\tilde{\kappa}_{E}-\tilde{\kappa}_{E} \star \tilde{\gamma}=0
$$

According to definition (14) of operator $\mathcal{L}$, if Loan Measure $\tilde{\kappa}_{E}$ satisfies equation in relation $(27)$, then, $\tilde{\kappa}_{E}$ is in $\operatorname{Ker}(\mathcal{L})$. Consequently, we have: 


$$
\operatorname{Ker}(\mathcal{L})=\left\{\tilde{\kappa}_{E} \in \mathcal{M}\left(\left[t_{\mathrm{I}}, \Theta_{\max }-\Theta_{\gamma}\right]\right), \tilde{\kappa}_{E}-\tilde{\kappa}_{E} \star \tilde{\gamma}=0\right\} .
$$

If Repayment Pattern Measure $\tilde{\gamma}$ is the Dirac mass $\delta_{0}$, then, relation $(28)$ gives that $\operatorname{Ker}(\mathcal{L})$ is equal to $\mathcal{M}\left(\left[t_{\mathrm{I}}, \Theta_{\max }-\Theta_{\gamma}\right]\right)$. In fact, $\delta_{0}$ is the identity element for the convolution operator. Thus linear operator $\mathcal{L}$ is not a one-to-one map if $\tilde{\gamma}=\delta_{0}$.

We distinguish four cases to prove the lemma:

\section{First case:}

In the first case, we will show that if Repayment Pattern Measure $\tilde{\gamma}$ is density measure satisfying relation (11), then, linear operator $\mathcal{L}$ given in relation (14) is a one-to-one map.

For any positive integer $n$, we define $\mathrm{V}_{n}$ the vector space of density measures such its densities are polynomial functions in $x$ of degree $n$ and restricted to interval $\left(t_{\mathrm{I}}, \Theta_{\max }-\Theta_{\gamma}\right)$. By density of space $\mathrm{V}_{m}$ for any integer $m$ in $\mathcal{M}\left(\left[t_{\mathrm{I}}, \Theta_{\max }-\Theta_{\gamma}\right]\right)$, we will show that for the following Repayment Pattern Measure $\tilde{\gamma} \in \mathrm{V}_{m}$

$$
\left(\frac{\sum_{i=0}^{i=m} \alpha_{i} t^{i}}{\sum_{i=0}^{i=m} \alpha_{i} \frac{\Theta_{\gamma}^{i+1}}{i+1}}\right) \times \mathbb{1}_{\left[0, \Theta_{\gamma}\right]}(t) d t
$$

that

$$
\operatorname{Ker}(\mathcal{L}) \cap \mathrm{V}_{n}=\{0\} .
$$

In order to show relation (30), we will show that the coefficients of density measure $\tilde{\kappa}_{E}$ are zero for each $\tilde{\kappa}_{E}$ in $\operatorname{Ker}(\mathcal{L}) \cap \mathrm{V}_{n}$. If $\tilde{\kappa}_{E} \in \operatorname{Ker}(\mathcal{L}) \cap \mathrm{V}_{n}$, then, for any $x \in\left(t_{\mathrm{I}}, \Theta_{\max }-\Theta_{\gamma}\right)$,

$$
\tilde{\kappa}_{E}=\left(\sum_{i=0}^{i=n} c_{i} x^{i}\right) \times \mathbb{1}_{\left[t_{\mathrm{I}}, \Theta_{\max }-\Theta_{\gamma}\right]}(x) d x,
$$

where $\left(c_{i}\right)_{0 \leq i \leq n}$ are its coefficients and

$$
\tilde{\kappa}_{E}-\tilde{\kappa}_{E} \star \tilde{\gamma}=0 .
$$

As $\tilde{\gamma}$ is given in relation (29), expression $\tilde{\kappa}_{E} \star \tilde{\gamma}$ can be simplified with using relation (31)

$$
\tilde{\kappa}_{E} \star \tilde{\gamma}=\frac{d x}{\sum_{i=0}^{i=m} \alpha_{i} \frac{\Theta_{\gamma}^{i+1}}{i+1}} \times \sum_{i=0}^{i=n} c_{i} \sum_{j=0}^{j=m} \alpha_{j} \int_{\left[t_{\mathrm{I}}, \Theta_{\max }-\Theta_{\gamma}\right] \cap\left[x-\Theta_{\gamma}, x\right]} y^{i}(x-y)^{j} d y
$$

As

$$
(x-y)^{j}=\sum_{p=0}^{p=j} C_{j}^{p}(x)^{j-p}(-y)^{p}
$$

expression (33) gives 


$$
\tilde{\kappa}_{E} \star \tilde{\gamma}=\frac{d x}{\sum_{i=0}^{i=m} \alpha_{i} \frac{\Theta_{\gamma}^{i+1}}{i+1}} \times \sum_{i=0}^{i=n} c_{i} \sum_{j=0}^{j=m} \alpha_{j}\left[\sum_{p=0}^{p=j} C_{j}^{p}(-1)^{p}(x)^{j-p} \times\left(\int_{\left[t_{\mathrm{I}}, \Theta_{\max }-\Theta_{\gamma}\right] \cap\left[x-\Theta_{\gamma}, x\right]} y^{i+p} d y\right)\right] .
$$

Now, on the one hand, we show that coefficients $\left(c_{i}\right)_{0<i<n}$ are zero, when $t_{\mathrm{I}}+\Theta_{\gamma} \leq \Theta_{\max }-\Theta_{\gamma}$. In this case, if $x$ is chosen outside the support of $\tilde{\kappa}_{E}$ such that $t_{\mathrm{I}}+\Theta_{\gamma} \leq \Theta_{\max }-\Theta_{\gamma}<x<\Theta_{\max }$, then, $\tilde{\kappa}_{E}$ is null. Thus, the intersection of intervals $\left[t_{\mathrm{I}}, \Theta_{\max }-\Theta_{\gamma}\right]$ and $\left[x-\Theta_{\gamma}, x\right]$ is interval $\left[\Theta_{\max }-\Theta_{\gamma}, x-\Theta_{\gamma}\right]$. Then, relations (32) and (35) are used with the fact that Loan Measure $\tilde{\kappa}_{E}$ is null in order to obtain the following equality:

$$
\sum_{i=0}^{i=n} c_{i} \sum_{j=0}^{j=m} \alpha_{j}\left[\sum_{p=0}^{p=j} \frac{C_{j}^{p}(-1)^{p}(x)^{j-p}}{i+p+1} \times\left(\left(\Theta_{\max }-\Theta_{\gamma}\right)^{i+p+1}-\left(x-\Theta_{\gamma}\right)^{i+p+1}\right)\right]=0 .
$$

From relation (36), we get a nul polynom on interval $\left(\Theta_{\max }-\Theta_{\gamma}, \Theta_{\max }\right)$ which has an non empty interior. Consequently, we showed that coefficients $\left(c_{i}\right)_{0 \leq i \leq n}$ are zero, when $t_{\mathrm{I}}+\Theta_{\gamma} \leq \Theta_{\max }-\Theta_{\gamma}$.

On other hand, we show that coefficients $\left(c_{i}\right)_{0 \leq i \leq n}$ are zero, when $\Theta_{\max }-\Theta_{\gamma}<t_{\mathrm{I}}+\Theta_{\gamma}$. Then, if $x$ is chosen outside the support of $\tilde{\kappa}_{E}$ such that $\Theta_{\max }-\Theta_{\gamma}<t_{\mathrm{I}}+\Theta_{\gamma}<x<\Theta_{\max }$, then, $\tilde{\kappa}_{E}$ is null. Thus, the intersection of intervals $\left[t_{\mathrm{I}}, \Theta_{\max }-\Theta_{\gamma}\right]$ and $\left[x-\Theta_{\gamma}, x\right]$ is interval $\left[\Theta_{\max }-\Theta_{\gamma}, x-\Theta_{\gamma}\right]$. We obtain the same null polynom in relation (36) on interval $\left(t_{\mathrm{I}}+\Theta_{\gamma}, \Theta_{\max }\right)$ which has an non empty interior.

Consequently, coefficients $\left(c_{i}\right)_{0<i<n}$ are zero. From this, we conclude that (30) is true for any integer $n$. The proof of the first case is achieved.

\section{Second case:}

In the second case, we will show that if Repayment Pattern Measure $\tilde{\gamma}$ is a density measure satisfying relation (11), then, linear operator $\mathcal{L}$ given by relation (14) is a one-to-one map for any Loan Measure $\tilde{\kappa}_{E}$ which is supposed a concentrated measure (as like as a combination of Dirac measures or a combination of Dirac measures multiplied by a continuons functions). In fact, since the convolution of $\tilde{\gamma}$ and $\tilde{\kappa}_{E}$ is concentrated measure, then, relation (27) gives that a density measure is equal to a concentrated measure. This equality is possible, if and only if, Loan Measure $\tilde{\kappa}_{E}$ is zero. Consequently, the proof of the second case is achieved.

\section{Third case:}

We show in the third case that if Repayment Pattern Measure $\tilde{\gamma}$ is a concentrated measure satisfying relation (11), i.e:

$$
\tilde{\gamma}=\sum_{j=1}^{j=m} e_{j} \delta_{a_{j}}
$$

Thus, for any Loan Measure $\tilde{\kappa}_{E}$ which is supposed a density measure, we have:

$$
\kappa_{E}(x)=\sum_{j=1}^{j=m} e_{j} \kappa_{E}\left(x+a_{j}\right) .
$$

Then Loan Density $\kappa_{E}$ is null. From this, linear operator $\mathcal{L}$ given by relation (14) is a one-to-one map for any Loan Density Measure $\tilde{\kappa}_{E}$.

\section{Fourth case:}


In the fourth case, we set the Repayment Pattern Measure $\tilde{\gamma}$ as a combination of m Dirac measures located at points $\left(a_{j}\right)_{1 \leq j \leq m}$ that is different from $\delta_{0}$ and satisfying relation (11) such that:

$$
\tilde{\gamma}=\sum_{j=1}^{j=m} e_{j} \delta_{a_{j}}
$$

We will show that the coefficients of concentrated measure $\tilde{\kappa}_{E}$ are zero for any Loan Measure $\tilde{\kappa}_{E}$ that can be written in the following form $\tilde{\kappa}_{E}=\sum_{i=1}^{i=n} f_{i} \delta_{b_{i}}$, where points $b_{i}$ are in $\left[t_{\mathrm{I}}, \Theta_{\max }-\Theta_{\gamma}\right]$ for i from 1 to $\mathrm{n}$. According to (27), we have:

$$
\sum_{i=0}^{i=n} f_{i} \times\left(\delta_{b_{i}}-\sum_{j=0}^{j=m} e_{j} \delta_{b_{i}+a_{j}}\right)=0
$$

In order to show that coefficients $\left(f_{i}\right)_{0 \leq i \leq n}$ are zero, we distinguish two situations of points $\left(a_{j}\right)_{1 \leq j \leq m}$. The first situation is when points $\left(a_{j}\right)_{1 \leq j \leq m}$ are not nulls. Thus, since points $\left(e_{j}\right)_{0 \leq j \leq m}$ are fixed points, relation (40) proves that coefficients $\left(f_{i}\right)_{0 \leq i \leq n}$ are zero. The second situation is when there exists $a_{k}=0$ for $1 \leq k \leq m$, then relation (40) gives

$$
\sum_{i=0}^{i=n} f_{i} \times\left(\left(1-e_{k}\right) \delta_{b_{i}}-\sum_{j=0, j \neq k}^{j=m} e_{j} \delta_{b_{i}+a_{j}}\right)=0
$$

Since Repayment Pattern Measure $\tilde{\gamma}$ is different from $\delta_{0}$, relation (41) proves that coefficients $\left(f_{i}\right)_{0 \leq i \leq n}$ are zero.

Consequently, the study of these four cases is achieving the proof of the lemma.

Lemma 3.5. Assuming that:

$$
\left\langle\tilde{\gamma}, t \mathbb{1}_{\mid\left[t_{\mathrm{I}}, \Theta_{\max }[\right.}\right\rangle \neq 0
$$

is achieved and if operator $\mathcal{L}$ given by relation (14) satisfies:

$$
\left\langle\mathcal{L}\left[\tilde{\kappa}_{E}\right]+\alpha \tilde{\mathrm{e}}_{\alpha}\left\langle\mathcal{L}\left[\tilde{\kappa}_{E}\right], \mathrm{e}_{-\alpha \mid\left[t_{\mathrm{I}}, t[\right.}\right\rangle, \mathbb{1}_{\mid\left[t_{\mathrm{I}}, \Theta_{\max } \mid\right.}\right\rangle=0,
$$

then, $\mathcal{F}\left(\tilde{\kappa}_{E}\right) \in \mathbb{L}^{\infty}(\mathbb{R})$ and is such that

$$
\lim _{\xi \rightarrow 0} \mathcal{F}\left(\tilde{\kappa}_{E}\right)(\xi)=-\frac{\left\langle\mathcal{L}\left[\tilde{\kappa}_{E}\right]+\alpha \tilde{\mathrm{e}}_{\alpha}\left\langle\mathcal{L}\left[\tilde{\kappa}_{E}\right], \mathrm{e}_{-\alpha \mid\left[t_{\mathrm{I}}, t\right]}\right\rangle, t \mathbb{1}_{\mid\left[t_{\mathrm{I}}, \Theta_{\max }[\right.}\right\rangle}{\left\langle\tilde{\gamma}, t \mathbb{1}_{\mid\left[t_{\mathrm{I}}, \Theta_{\max }[\right.}\right\rangle} .
$$

If $\mathcal{L}$ does not satisfy the equality in relation $(43)$, then, $\mathcal{F}\left(\tilde{\kappa}_{E}\right)$ has an infinite limit in 0.

Proof. As $\mathcal{L}\left[\tilde{\kappa}_{E}\right] \in \mathcal{M}\left(\left[t_{\mathrm{I}}, \Theta_{\max }\right]\right)$, we get:

$$
\mathcal{L}\left[\tilde{\kappa}_{E}\right]+\alpha \tilde{\mathrm{e}}_{\alpha}\left\langle\mathcal{L}\left[\tilde{\kappa}_{E}\right], \mathrm{e}_{-\alpha \mid\left[t_{\mathrm{I}}, t[\right.}\right\rangle \in \mathcal{M}\left(\left[t_{\mathrm{I}}, \Theta_{\max }\right]\right)
$$

Function $\mathcal{F}\left(\mathcal{L}\left[\tilde{\kappa}_{E}\right]+\alpha \tilde{\mathrm{e}}_{\alpha}\left\langle\mathcal{L}\left[\tilde{\kappa}_{E}\right], \mathrm{e}_{-\alpha \mid\left[t_{\mathrm{I}}, t[\right.}\right\rangle\right)$ is expanded in 0 until the order 1 with using the Taylor expansion of exponential function $e^{-i y \xi}$ in 0 until the order 1 to get: 


$$
\begin{aligned}
\mathcal{F}\left(\mathcal{L}\left[\tilde{\kappa}_{E}\right]+\alpha \tilde{\mathrm{e}}_{\alpha}\left\langle\mathcal{L}\left[\tilde{\kappa}_{E}\right], \mathrm{e}_{-\alpha \mid\left[t_{I}, t\right]}\right\rangle\right)(\xi) & =\left\langle\mathcal{L}\left[\tilde{\kappa}_{E}\right]+\alpha \tilde{\mathrm{e}}_{\alpha}\left\langle\mathcal{L}\left[\tilde{\kappa}_{E}\right], \mathrm{e}_{-\alpha \mid\left[t_{I}, t\right]}\right\rangle, \mathbb{1}_{\mid\left[t_{\mathrm{I}}, \Theta_{\max } \mid\right.}\right\rangle \\
& -i \xi\left\langle\mathcal{L}\left[\tilde{\kappa}_{E}\right]+\alpha \tilde{\mathrm{e}}_{\alpha}\left\langle\mathcal{L}\left[\tilde{\kappa}_{E}\right], \mathrm{e}_{-\alpha \mid\left[t_{I}, t \mid\right.}\right\rangle, t \mathbb{1}_{\mid\left[t_{\mathrm{I}}, \Theta_{\max } \mid\right.}\right\rangle+O\left(\xi^{2}\right)
\end{aligned}
$$

Since operator $\mathcal{L}$ satisfies equality in relation (43), relation (46) gives:

$$
\mathcal{F}\left(\mathcal{L}\left[\tilde{\kappa}_{E}\right]+\alpha \tilde{\mathrm{e}}_{\alpha}\left\langle\mathcal{L}\left[\tilde{\kappa}_{E}\right], \mathrm{e}_{-\alpha \mid\left[t_{\mathrm{I}}, t\right]}\right\rangle\right)(\xi)=-i \xi\left\langle\mathcal{L}\left[\tilde{\kappa}_{E}\right]+\alpha \tilde{\mathrm{e}}_{\alpha}\left\langle\mathcal{L}\left[\tilde{\kappa}_{E}\right], \mathrm{e}_{-\alpha \mid\left[t_{\mathrm{I}}, t\right]}\right\rangle, t \mathbb{1}_{\mid\left[t_{\mathrm{I}}, \Theta_{\max }[\right.}\right\rangle+O\left(\xi^{2}\right)
$$

According to (11), function $1-\mathcal{F}(\tilde{\gamma})$ is Taylor expanded in 0 until the order 1 to obtain:

$$
1-\mathcal{F}(\tilde{\gamma})(\xi)=i \xi\left\langle\tilde{\gamma}, t \mathbb{1}_{\mid\left[t_{\mathrm{I}}, \Theta_{\max } \mid\right.}\right\rangle+O\left(\xi^{2}\right) .
$$

According to relations (19), (47) and (48), we get equality (44).

As $\mathcal{L}\left[\tilde{\kappa}_{E}\right]$ and $\tilde{\gamma}$ are integrables on their supports, the numerator and the denominator of right term in relation (44) are finites. From this and according to relation (42), function $\mathcal{F}\left(\tilde{\kappa}_{E}\right)$ admits a finite limit at 0 . It is concluded $\mathcal{F}\left(\tilde{\kappa}_{E}\right)$ is in space $\mathbb{L}^{\infty}(\mathbb{R})$.

On other hand, if equality in relation (43) is not satisfied, then, according to relations (19), (46) and (48), $\mathcal{F}\left(\tilde{\kappa}_{E}\right)$ has an infinite limit at 0 .

From this, the proof of lemma is achieved.

Lemma 3.6. Under assumptions (11), (24), (43) and if operator $\mathcal{L}$ given by relation (14) satisfies:

$$
\mathcal{F}\left(\mathcal{L}\left[\tilde{\kappa}_{E}\right]\right) \in \mathbb{L}^{2}\left(\left[t_{\mathrm{I}}, \Theta_{\max }\right]\right),
$$

then, $\mathcal{F}\left(\tilde{\kappa}_{E}\right) \in \mathbb{L}^{2}(\mathbb{R})$.

Proof. According to (49), we get:

$$
\mathcal{F}\left(\mathcal{L}\left[\tilde{\kappa}_{E}\right]+\alpha \tilde{\mathrm{e}}_{\alpha}\left\langle\mathcal{L}\left[\tilde{\kappa}_{E}\right], \mathrm{e}_{-\alpha \mid\left[t_{\mathrm{I}}, t\right]}\right\rangle\right) \in \mathbb{L}^{2}(\mathbb{R}) .
$$

We notice that for any positive $\epsilon$, the product of functions in $\mathbb{L}^{\infty}((-\infty,-\epsilon) \cup(\epsilon,+\infty))$ and in $\mathbb{L}^{2}(\mathbb{R})$ is a function in $\mathbb{L}^{2}((-\infty,-\epsilon) \cup(\epsilon,+\infty))$. From relations (19), (50) and according to the assumption of $\tilde{\gamma}$ given in relation $(24)$, we have:

$$
\exists \epsilon>0, \mathcal{F}\left(\tilde{\kappa}_{E}\right) \in \mathbb{L}^{2}((-\infty,-\epsilon) \cup(\epsilon,+\infty)) .
$$

According to Lemma $3.5, \mathcal{F}\left(\tilde{\kappa}_{E}\right)$ admits a finite limit at point $\xi=0$. Consequently, we have the following result:

$$
\exists \epsilon>0, \mathcal{F}\left(\tilde{\kappa}_{E}\right) \in \mathbb{L}^{2}([-\epsilon, \epsilon]) .
$$

From this and using relation (51), the lemma is proved.

Proof of Theorem 3.3. We notice that Repayment Pattern Measure $\tilde{\gamma}$ equals to Dirac mass $\delta_{0}$ doesn't satisfy relation (24). From Lemma 3.4, we obtain the uniqueness of $\tilde{\kappa}_{E}$, achieving the proof of the theorem. 


\section{A concentration method of the model}

We developped a new approach of concentrating a measure to enrich the model. This approach is allowing to generate the result measure which is called the concentrated measure. Indeed, concentrate Repayment Measure $\tilde{\rho}_{\mathcal{K}}$ can be interpreted from the financial slant as follow. It means that Repayment amount presented by $\tilde{\rho}_{\mathcal{K}}$ is associated with localized actions or payments at differents instants in order to express how these payments are distributed at these instants. Thus, the total repayment amount is equal to the sum of these amounts. This equality defines a principle of conservation that we explain it below.

For any integer $n$ strictly greater than 1 , we define $J$ the set of integers between 1 and $n$, i.e $J=\llbracket 1 ; n \rrbracket$. To concentrate a given measure $m$, we assume that its integral over $\mathbb{R}$ is finite

$$
\int_{-\infty}^{+\infty} m<\infty
$$

The concentrated measure of measure $m$ is defined as a sum of Dirac masses $\left(M_{i}\right)_{i \in J}$ located in given positions. The basic idea of concentrating the measure $m$ is to determine the adequate partition of unity which is defined by a set of nonnegative compactly supported functions $\left(\phi_{i}\right)_{i \in J}$. Afterwards, measures defined by a multiplication of measure $m$ by functions $\left(\phi_{i}\right)_{i \in J}$ are integrated in order to compute Dirac masses $\left(M_{i}\right)_{i \in J}$.

To construct this partition of unity, we need three quantities. The first one is the set of intervals $\left(\left[d_{i}, f_{i}\right]\right)_{i \in J}$, where $d_{i}<f_{i}$ for each $i \in J$. In order that the intersection of intervals $\left[d_{i}, f_{i}\right]$ and $\left[d_{i+1}, f_{i+1}\right]$ is non empty for each $i \in J \backslash\{n\}$, we state the following assumption:

$$
\forall i \in J \backslash\{n\}, d_{i+1}<f_{i} .
$$

The second one is the fuzzy index $F I$ which influences on calculated Dirac masses $\left(M_{i}\right)_{i \in J}$. The third one is the set of positives values $\left(C L_{i}\right)_{i \in J}$ used to balance Dirac masses $\left(M_{i}\right)_{i \in J}$ on each intersection of intervals $\left[d_{i}, f_{i}\right]$. We notice that the quantities $\left(\left[d_{i}, f_{i}\right]\right)_{i \in J}$ and $F I$ are required and the quantity $\left(C L_{i}\right)_{i \in J}$ is optional. Fuzzy index $F I$ is assumed not great enough.

After definning needed quantities to concentrate measure $m$, partition of unity function $\phi_{i}$ is supposed with support being $\left[d_{i}, f_{i}\right]$ for each $i \in J$

$$
\forall i \in J, \operatorname{Supp}\left(\phi_{i}\right)=\left[d_{i}, f_{i}\right]
$$

This allows us to define Dirac masses $\left(M_{i}\right)_{i \in J}$ as follows:

$$
\forall i \in J, M_{i}=\int_{d_{i}}^{f_{i}} m \times \phi_{i},
$$

where, a measure $m \times \phi_{i}$ is the product of measure $m$ by function $\phi_{i}$.

Now, we show the principle of conservation of masses which states that the sum of Dirac masses $\left(M_{i}\right)_{i \in J}$ and the total mass of measure $m$ presented by its integral are equals. Since functions $\left(\phi_{i}\right)_{i \in J}$ form a partition of unity, we have $\sum_{i \in J} \phi_{i}=1$ which is multiplied by measure $m$ to get:

$$
m=\sum_{i \in J} m \times \phi_{i}
$$

By integrating over $\mathbb{R}$ measure $m$ given by relation (57) and by permuting a finite sum and finite integral, we get the following relation:

$$
\int_{-\infty}^{+\infty} m=\sum_{i \in J} M_{i}
$$


which expresses that the total mass $\int_{-\infty}^{+\infty} m$ is the sum of the Dirac masses $\left(M_{i}\right)_{i \in J}$. It is concluded that the principle of conservation is proved.

\subsection{Construction of the partition of unity}

It is difficult to build explicitly the partition of unity in our model. Consequently, this partition of unity is determined with approximated numerical method in two steps. The first step is an initialization and the second step is an iteration. We explain these steps as follows:

\subsubsection{Initialization step}

The aim of the initialization step is to initialize positives functions $\left(\phi_{i}^{0}\right)_{i \in J}$ defined on $\mathbb{R}$ which are not necessary a partition of unity. We notice that the expression of functions $\left(\phi_{i}^{0}\right)_{i \in J}$ depends on the fuzzy index $F I$ and on its support which is given by following relation:

$$
\forall i \in J, \operatorname{Supp}\left(\phi_{i}^{0}\right)=\left[d_{i}, f_{i}\right] .
$$

We determine functions $\left(\phi_{i}^{0}\right)_{i \in J}$ in three cases of the fuzzy index $F I$ which are $F I=0, F I=1$ and $F I \geq 2$.

If $F I=0$, function $\phi_{i}^{0}$ (see Figure 1 ) is a constant piecewise function, which is 1 over $\left[d_{i}, f_{i}\right]$ for each $i \in J$.

$$
\forall i \in J, \phi_{i}^{0}(x)=\mathbb{1}_{\left[d_{i}, f_{i}\right]}(x)
$$

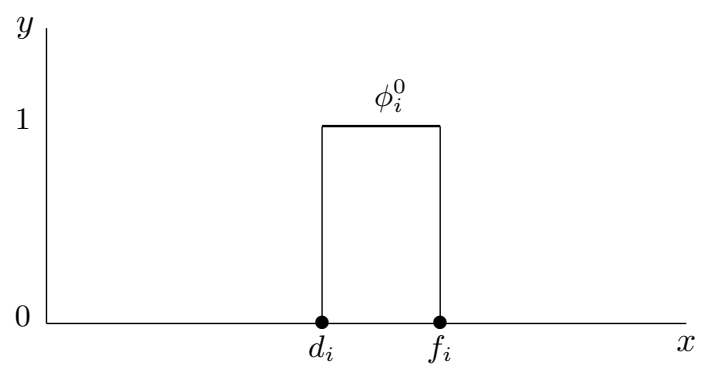

Figure 1: Definition of functions $\left(\phi_{i}^{0}\right)_{i \in J}$ when $F I=0$

If $F I=1$, we define the point $c_{i}$ as the mid-point of the line segment $\left[d_{i}, f_{i}\right]$. Then, function $\phi_{i}^{0}$ (see Figure 2) is an affine piecewise function on intervals $\left[d_{i}, c_{i}\right]$ and $\left[c_{i}, f_{i}\right]$ for each $i \in J$, which is defined as follows. The restriction of function $\phi_{i}^{0}$ on interval $\left[d_{i}, c_{i}\right]$ is an affine function which is 0 at point $d_{i}$ and is 1 at point $c_{i}$ for each $i \in J$. The restriction of function $\phi_{i}^{0}$ on interval $\left[c_{i}, f_{i}\right]$ is an affine function which is 1 at point $c_{i}$ and is 0 at point $f_{i}$ for each $i \in J$.

$$
\forall i \in J, \phi_{i}^{0}(x)=\frac{x-d_{i}}{c_{i}-d_{i}} \mathbb{1}_{\left[d_{i}, c_{i}[\right.}(x)+\frac{x-f_{i}}{c_{i}-f_{i}} \mathbb{1}_{\left[c_{i}, f_{i}\right]}(x) .
$$

If $F I \geq 2$, function $\phi_{i}^{0}$ (see Figure 3 ) is an affine piecewise function on intervals $\left[d_{i}^{\prime}, c_{i}^{\prime}\right]$ and $\left[c_{i}^{\prime}, f_{i}^{\prime}\right]$ for each $i \in J$, where the points $d_{i}^{\prime}$ and $f_{i}^{\prime}$ are to be determined respectively in terms of values $\left(d_{i}\right)_{i \in J}$ and $\left(f_{i}\right)_{i \in J}$ and where the point $c_{i}^{\prime}$ is the mid-point of the line segment $\left[d_{i}^{\prime}, f_{i}^{\prime}\right]$.

Now we will determine the points $d_{i}^{\prime}$ and $f_{i}^{\prime}$ for each $i \in J$ as follows. We define each value $d_{i}^{\prime}$ by a value $\frac{d_{j}}{2^{F I}}$, where point $d_{j}$ is the left neighborhood of the point $d_{i}$ such that a distance $d_{i}-d_{j}$ is strictly positive and is minimized. If there is any point strictly inferior than $d_{i}$, we take $d_{j}=d_{i}$. Formally, points $\left(d_{i}^{\prime}\right)_{i \in J}$ are defined as follows: 


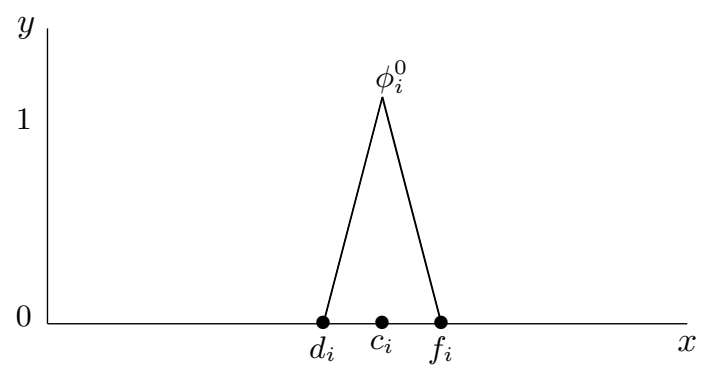

Figure 2: Definition of functions $\left(\phi_{i}^{0}\right)_{i \in J}$ when $F I=1$

$$
\forall i \in J, d_{i}^{\prime}=\left\{\frac{d_{j}}{2^{F I}}, \min _{d_{j}}\left(d_{i}-d_{j}\right)>0\right\} \cup\left\{\frac{d_{i}}{2^{F I}}\right\}
$$

Similarly for the point $d_{i}^{\prime}$, the point $f_{i}^{\prime}$ is determined by a value $\frac{f_{j}}{2^{F T}}$, where point $f_{j}$ is the right neighborhood of the point $f_{i}$ such that a distance $f_{j}-f_{i}$ is strictly positive and is minimized. If there is any point strictly superior than $f_{i}$, we take $f_{j}=f_{i}$. Formally, points $\left(f_{i}^{\prime}\right)_{i \in J}$ are defined as follows:

$$
\forall i \in J, f_{i}^{\prime}=\left\{\frac{f_{j}}{2^{F I}}, \min _{f_{j}}\left(f_{j}-f_{i}\right)>0\right\} \cup\left\{\frac{f_{i}}{2^{F I}}\right\} .
$$

Since points $\left(d_{i}^{\prime}\right)_{i \in J}$ and $\left(f_{i}^{\prime}\right)_{i \in J}$ are given respectively by relations (62) and (63), we determine function $\phi_{i}^{0}$ on interval $\left[d_{i}^{\prime}, f_{i}^{\prime}\right]$ for each $i \in J$ when $F I \geq 2$ as follows. The restriction of function $\phi_{i}^{0}$ on interval $\left[d_{i}^{\prime}, c_{i}^{\prime}\right]$ is an affine function which is 0 at point $d_{i}^{\prime}$ and is 1 at point $c_{i}^{\prime}$ for each $i \in J$. The restriction of function $\phi_{i}^{0}$ on interval $\left[c_{i}^{\prime}, f_{i}^{\prime}\right]$ is an affine function which is 1 at point $c_{i}^{\prime}$ and is 0 at point $f_{i}^{\prime}$ for each $i \in J$. Formally, functions $\left(\phi_{i}^{0}\right)_{i \in J}$ are defined as follows:

$$
\forall i \in J, \phi_{i}^{0}(x)=\frac{x-d_{i}^{\prime}}{c_{i}^{\prime}-d_{i}^{\prime}} \mathbb{1}_{\left[d_{i}^{\prime}, c_{i}^{\prime}[\right.}(x)+\frac{x-f_{i}^{\prime}}{c_{i}^{\prime}-f_{i}^{\prime}} \mathbb{1}_{\left[c_{i}^{\prime}, f_{i}^{\prime}\right]}(x)
$$

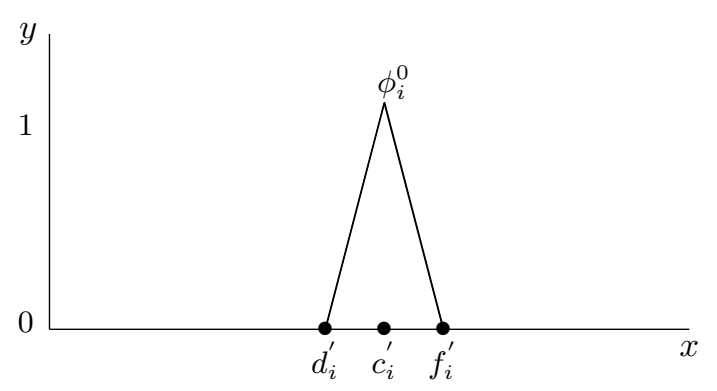

Figure 3: Definition of functions $\left(\phi_{i}^{0}\right)_{i \in J}$ when $F I \geq 2$

\subsubsection{Iteration step}

The aim of the iteration step is to compute functions $\left(\phi_{i}\right)_{i \in J}$ which form a partition of unity in terms of initialized functions $\left(\phi_{i}^{0}\right)_{i \in J}$ given by relation (60) if $F I=0$ or by relation (61) if $F I=1$ or by relation (64) if $F I \geq 2$. Since $\left(C L_{i}\right)_{i \in J}$ is an optional parameter in our modeling, functions $\left(\phi_{i}\right)_{i \in J}$ are determined in two cases. The first case is when we don't utilize $\left(C L_{i}\right)_{i \in J}$. The second case is when we utilize $\left(C L_{i}\right)_{i \in J}$. In the first case, functions $\left(\phi_{i}\right)_{i \in J}$ are defined as follows: 


$$
\forall i \in J, \forall x \in \bigcup_{i \in J}\left[d_{i}, f_{i}\right], \phi_{i}(x)=\frac{\phi_{i}{ }^{0}(x)}{\sum_{i \in J} \phi_{i}{ }^{0}(x)} .
$$

In the second case, functions $\left(\phi_{i}\right)_{i \in J}$ are defined as follows:

$$
\forall i \in J, \forall x \in \bigcup_{i \in J}\left[d_{i}, f_{i}\right], \phi_{i}(x)=\frac{C L_{i} \times \phi_{i}{ }^{0}(x)}{\sum_{i \in J} C L_{i} \times \phi_{i}{ }^{0}(x)} .
$$

Now, we can verify that functions $\left(\phi_{i}\right)_{i \in J}$ given by relation (65) or by relation (66) form a partition of unity. Indeed, functions $\left(\phi_{i}\right)_{i \in J}$ are positives because of the positivity of functions $\left(\phi_{i}^{0}\right)_{i \in J}$ and of values $\left(C L_{i}\right)_{i \in J}$ and the sum of functions $\left(\phi_{i}\right)_{i \in J}$ is 1 satisfying following equality:

$$
\sum_{i \in J} \phi_{i}(x)=1 .
$$

\subsection{Examples of the partition of unity}

In order to illustrate the construction of the partition of unity developped in subsection 4.1, we draw examples of functions $\phi_{1}$ and $\phi_{2}$ in Figures 4 and 5 which are defined on $\mathbb{R}$ with support being respectively $[0,6]$ and $[2,4]$, i.e $d_{1}=0, f_{1}=6, d_{2}=2$ and $f_{2}=4$. In addition, since the fuzzy index $F I$ is equal to 0 in simulation of Figure 4, functions $\phi_{1}$ and $\phi_{2}$ are piecewise constants functions and are computed with relation (65) or relation (66).

The left picture of Figure 4 shows the partition of unity formed by functions $\phi_{1}$ and $\phi_{2}$ which are computed with relation (65). The top diagram presents function $\phi_{1}$ which is equal to $\frac{1}{2}$ between instants 2 and 4 and to 1 between instants 0 and 2 and between instants 4 and 6 and to 0 elsewhere. The middle diagram presents function $\phi_{2}$ which is equal to $\frac{1}{2}$ between instants 2 and 4 and to 0 elsewhere. The bottom diagram shows that function $\phi_{1}+\phi_{2}$ is equal to 1 between instants 0 and 6 . This example illustrates that formula (65) computes correctly the partition of unity.

The right picture of Figure 4 shows the partition of unity formed by functions $\phi_{1}$ and $\phi_{2}$ which are computed with relation (66) using coefficients $C L_{1}=1$ and $C L_{2}=10$. The top diagram presents function $\phi_{1}$ which is equal to $\frac{1}{11}$ between instants 2 and 4 and to 1 between instants 0 and 2 and between instants 4 and 6 . The middle diagram presents function $\phi_{2}$ which is equal to $\frac{10}{11}$ between instants 2 and 4 and to 0 elsewhere. The bottom diagram shows that the sum of functions $\phi_{1}$ and $\phi_{2}$ is equal to 1 between instants 0 and 6 . This example illustrates that formula (66) computes correctly the partition of unity.

Now, if the fuzzy index $F I$ is equal to 1 in simulation of Figure 5 , functions $\phi_{1}$ and $\phi_{2}$ are piecewise rational fractions over time periods $[2,3]$ and $[3,4]$ that are in form of fractions of affines functions.

The left picture of Figure 5 shows the partition of unity formed by functions $\phi_{1}$ and $\phi_{2}$ which are computed with relation (65). The top diagram presents function $\phi_{1}$ which is a rational function over time periods $[2,3]$ and $[3,4]$ and is equal to $\frac{1}{2}$ at instant 3 . Function $\phi_{1}$ is equal to 1 over time periods $[0,2]$ and $[4,6]$. The middle diagram presents function $\phi_{2}$ which is a rational function over time periods $[2,3]$ and $[3,4]$ and is equal to $\frac{1}{2}$ at instant 3 . Function $\phi_{2}$ is null over time periods $[0,2]$ and $[4,6]$. The bottom diagram shows that function $\phi_{1}+\phi_{2}$ is equal to 1 between instants 0 and 6 . Consequently, relation (65) computes correctly the partition of unity.

The right picture of Figure 5 shows the partition of unity formed by functions $\phi_{1}$ and $\phi_{2}$ which are computed with relation (66) using coefficients $C L_{1}=1$ and $C L_{2}=10$. The top diagram presents 
function $\phi_{1}$ and the middle diagram presents function $\phi_{2}$. Functions $\phi_{1}$ and $\phi_{2}$ are rational functions over time periods $[2,3]$ and $[3,4]$. Function $\phi_{1}$ is equal to 1 over time periods $[0,2]$ and $[4,6]$, whereas function $\phi_{2}$ is null over time periods $[0,2]$ and $[4,6]$. The bottom diagram shows that function $\phi_{1}+\phi_{2}$ is equal to 1 between instants 0 and 6 .

\subsection{Financial simulation}

We will justify, in this section, relation (56), that computes Dirac masses of the concentrated measure. We will adopt both mathematical and financial point of view.

The left picture of Figure 6 represents two concentrated measures of Loan Measure $\tilde{\kappa}_{E}$. This Loan Measure $\tilde{\kappa}_{E}$ is shown in the top diagram which is a piecewise function that is equal to 1 between instants 0 and 0.5 , to 4 between instants 1 and 2 , to 6 between instants 3 and 4 , to 8 between instants 4 and 5 , to 3 between instants 5 and 5.5 and to 0 elsewhere. The first concentrated measure (see middle diagram) is computed with fuzzy index $F I$ equals to 0 . The second concentrated measure (see bottom diagram) is computed with fuzzy index $F I$ equals to 0 and with coefficients $C L_{1}=1$ and $C L_{2}=10$. We precise that we use the partition of unity presented at the left picture and at the right picture of Figure 4 to compute respectively the first concentrated measure and the second concentrated measure.

The measure drawn in the middle diagram is computed with formula (56) and is a combination of two Dirac masses (the first Dirac mass has the mass 17 at time 2.5 and the second Dirac mass has the mass 3 at time 3.5). This simulation can be interpreted from the financial slant as follows. It means that the loan is shared into two pieces, the first one consists in borrowing 17 at time 2.5 and the second one consists in borrowing 3 at time 3.5. Thus, the total borrowed amount is equal to $17+3=20$. This makes that formula (58) is homogeneous with regard to this simulation.

The bottom diagram shows the concentrated measure which is computed with relation (56). It is a combination of two Dirac masses (the first Dirac mass has the mass $\frac{160}{11}$ at time 2.5 and the second Dirac mass has the mass $\frac{60}{11}$ at time 3.5). The meaning of this simulation can be given as follows. It means that the loan is shared into two pieces, the first one consists in borrowing $\frac{160}{11}$ at time 2.5 and the second one consists in borrowing $\frac{60}{11}$ at time 3.5. This loan is the localized loan of $\tilde{\kappa}_{E}$ at instants 2.5 and 3.5. It means that the total borrowed amount is made of amount $\frac{160}{11}$ at times 2.5 and amount $\frac{60}{11}$ at time 3.5, which is equal to $\frac{160}{11}+\frac{60}{11}=20$. Consequently, since the amount borrowed associated to Loan Measure $\tilde{\kappa}_{E}$ between times 0 and 6 is 20 , relation (58) is homogeneous.

In order to illustrate formulas (56) and (58), in the right picture of Figure 6, we draw examples of concentrated measures of $\tilde{\kappa}_{E}$ with fuzzy index $F I$ equals to 1 . This picture shows three measures, the first measure is Loan Measure $\tilde{\kappa}_{E}$ shown in the top diagram which is the same as previously (see top diagram of the left picture). The other measures are the concentrated ones of $\tilde{\kappa}_{E}$. We use the partition of unity presented at the left picture and at the right picture of Figure 5 to compute respectively the first concentrated measure (see middle diagram) and the second concentrated measure (see bottom diagram).

These two concentrated measures are computed with formula (56). The first concentrated measure in the middle diagram is $17.5 \delta_{t=2.5}+2.5 \delta_{t=3.5}$. This illustrates that the loan gives the way that an amount 17.5 is borrowed at time 2.5 and an amount 2.5 is borrowed at time 3.5 . It means that the total borrowed amount is equal to 20 . The second concentrated measure in the bottom diagram is $15.2 \delta_{t=2.5}+4.8 \delta_{t=3.5}$. This concentrated measure means that an amount 15.2 is borrowed at time 2.5 and an amount 4.8 is borrowed at time 3.5. It means that the total borrowed amount is equal to 20. As the amount borrowed associated to Loan Measure $\tilde{\kappa}_{E}$ between times 0 and 6 is equal to 20, formula (58) is correct. It is concluded that this financial simulation using concentrated measures gives realistic results. 

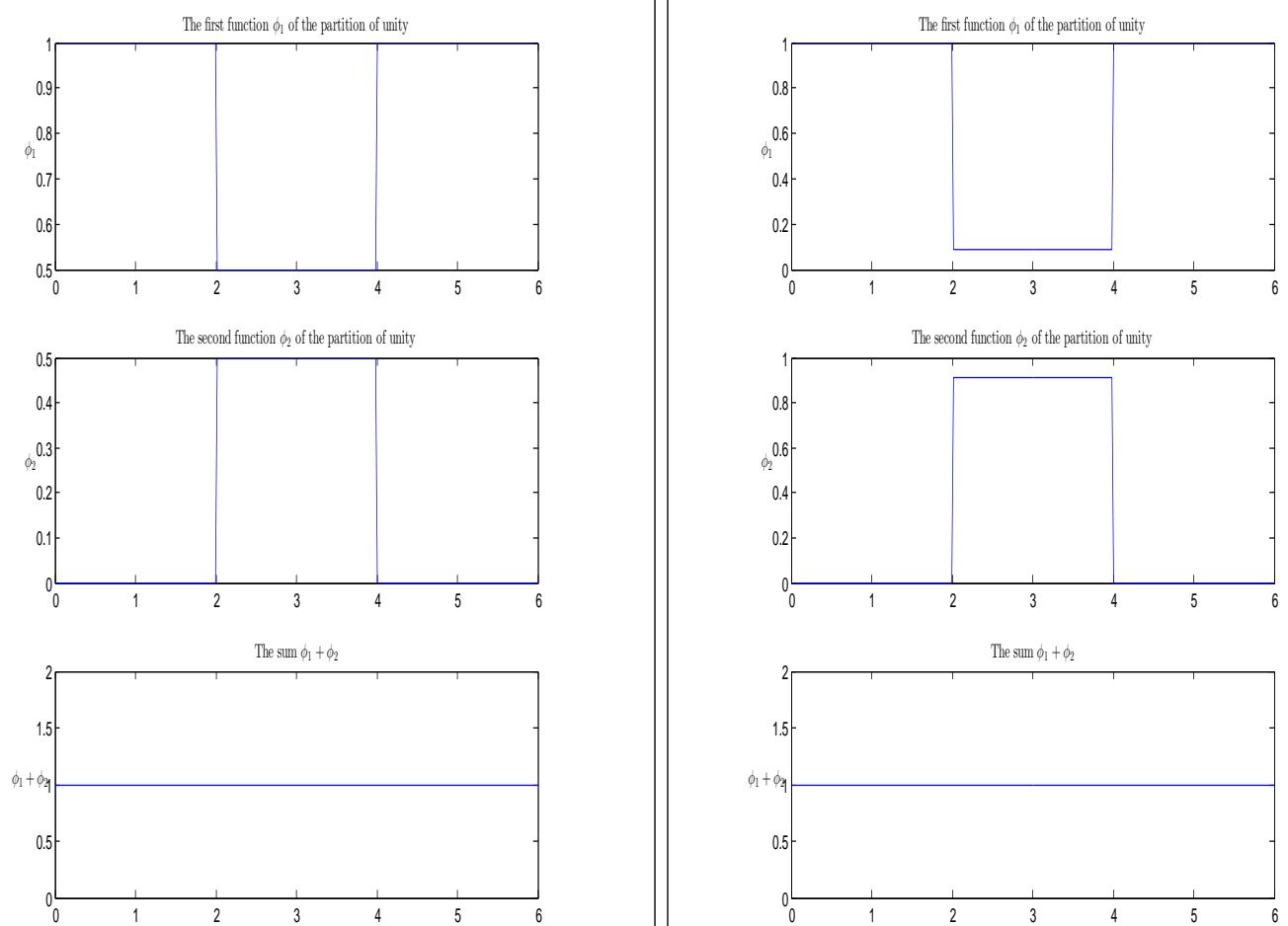

Figure 4: In each of the two pictures, the fuzzy index $F I$ is equal to 0 . In the left picture, function $\phi_{1}$ is represented at the top, function $\phi_{2}$ in the middle which are computed with relation (65) and their sum $\phi_{1}+\phi_{2}$ at the bottom. In the right picture, function $\phi_{1}$ is represented at the top, function $\phi_{2}$ in the middle which are computed with relation (66) with coefficients $C L_{1}=1$ and $C L_{2}=10$ and the sum $\phi_{1}+\phi_{2}$ at the bottom. 

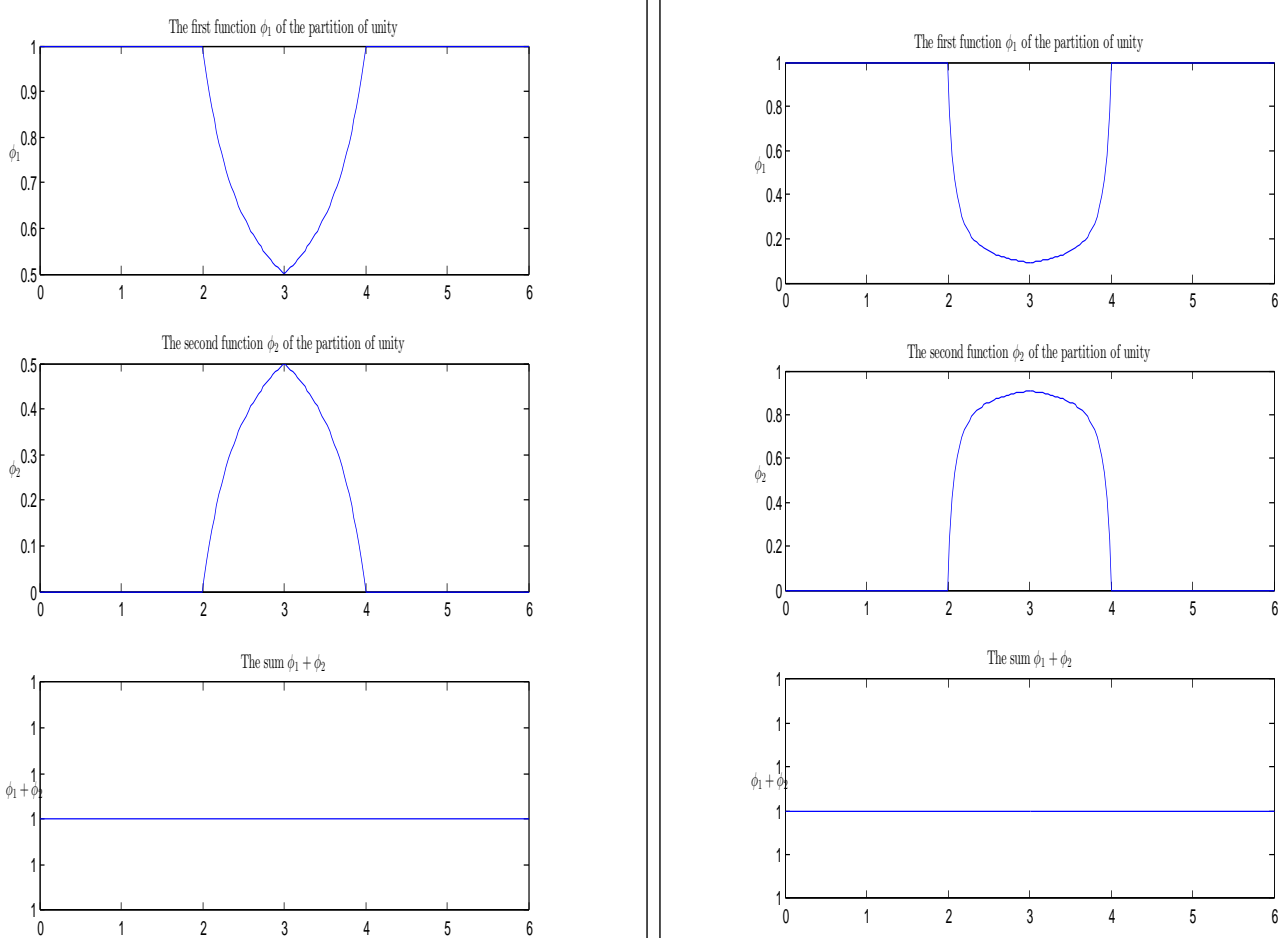

Figure 5: In each of the two pictures, the fuzzy index $F I$ is equal to 1 . In the left picture, function $\phi_{1}$ is represented at the top, function $\phi_{2}$ in the middle which are computed with relation (65) and their sum $\phi_{1}+\phi_{2}$ at the bottom. In the right picture, function $\phi_{1}$ is represented at the top, function $\phi_{2}$ in the middle which are computed with relation (66) with coefficients $C L_{1}=1$ and $C L_{2}=10$ and the sum $\phi_{1}+\phi_{2}$ at the bottom. 

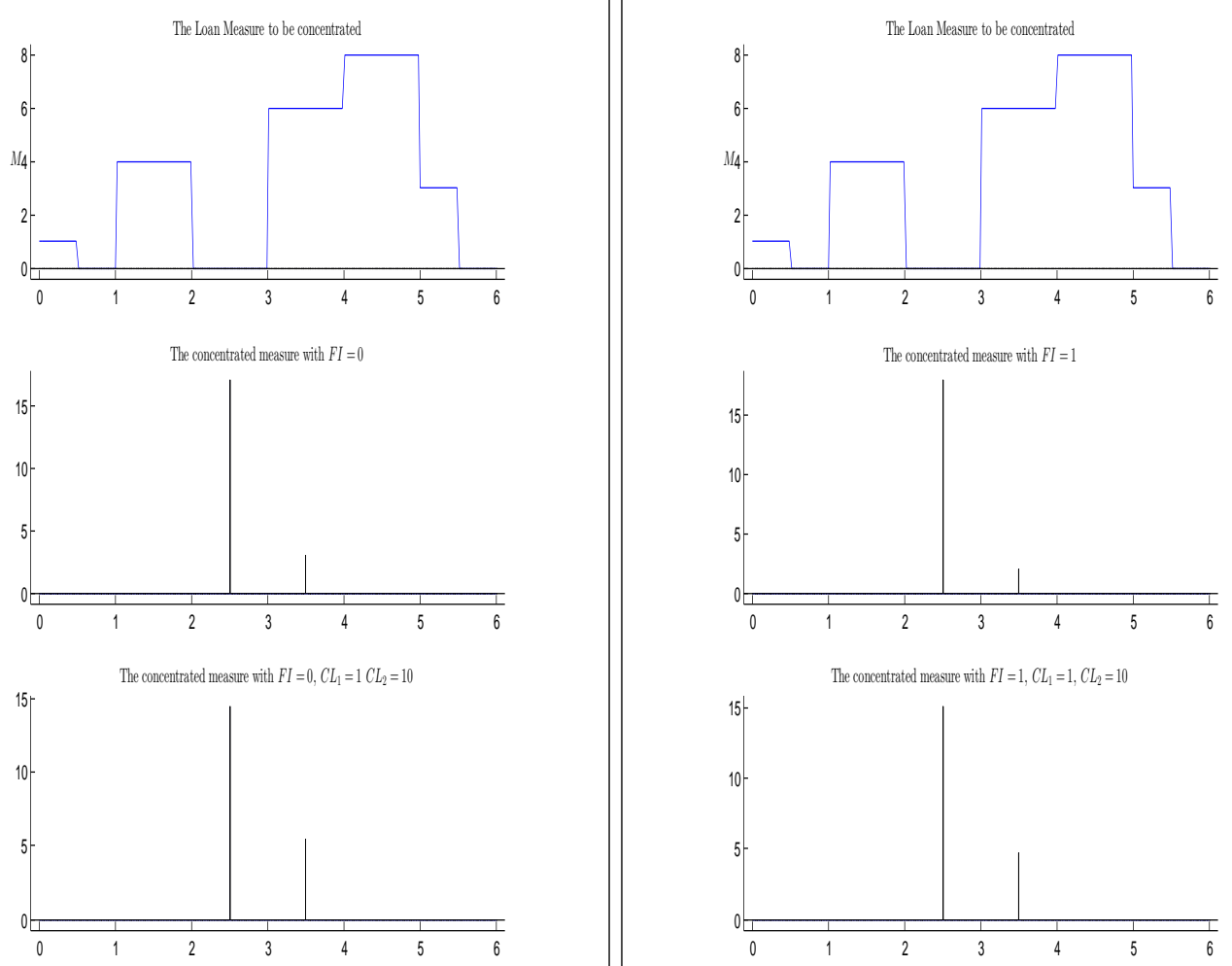

Figure 6: In each of the two pictures. In the left picture, fuzzy index FI equals to 0, Loan Measure $\tilde{\kappa}_{E}$ is represented at the top, in the middle the concentrated measure of $\tilde{\kappa}_{E}$ is computed with the partition of unity presented at the left picture of Figure 4, in the bottom the concentrated measure of $\tilde{\kappa}_{E}$ is computed with the partition of unity presented at the right picture of Figure 4 . In the right picture, fuzzy index $F I$ equals to $1, \tilde{\kappa}_{E}$ is represented at the top, in the middle the concentrated measure of $\tilde{\kappa}_{E}$ is computed with the partition of unity presented at the left picture of Figure 5, in the bottom the concentrated measure of $\tilde{\kappa}_{E}$ is computed with the partition of unity presented at the right picture of Figure 5 . 


\section{References}

[1] Carl Chiarella, Mark Craddock, Nadima El-Hassan, et al. The calibration of stock option pricing models using inverse problem methodology. QFRQ Research Papers, UTS Sydney, 2000.

[2] Herbert Egger and Heinz W Engl. Tikhonov regularization applied to the inverse problem of option pricing: convergence analysis and rates. Inverse Problems, 21(3):1027, 2005.

[3] Emmanuel Frénod and Tarik Chakkour. A continuous-in-time financial model. Mathematical Finance Letters, pages 1-36, ID2, 2016.

[4] Emmanuel Frénod, Pierre Menard, and Mohamad Safa. Optimal control of a continuous-in-time financial model. Mathematical Modelling and Numerical Analysis, 2013. Manuscript in revision.

[5] Emmanuel Frénod, Pierre Menard, and Mohamad Safa. Two optimization problems using a continuous-in-time financial model. Journal of Industrial and Management Optimization, 2014. Manuscript in revision.

[6] Emmanuel Frńod and Mohamad Safa. Continuous-in-time financial model for public communities. volume 45, pages 158-167. EDP Sciences, 2014.

[7] Robert C Merton. Theory of finance from the perspective of continuous time. Journal of Financial and Quantitative Analysis, 10(04):659-674, 1975.

[8] Robert C Merton and Paul Anthony Samuelson. Continuous-Time Finance. Blackwell Boston, 1st edition, 1992.

[9] Suresh M Sundaresan. Continuous-time methods in finance: A review and an assessment. The Journal of Finance, 55(4):1569-1622, 2000. 